\title{
Clinical significance of the estrogen-modifying enzymes steroid sulfatase and estrogen sulfotransferase in epithelial ovarian cancer
}

\author{
FELICITAS MUNGENAST $^{1 *}$, STEFANIE AUST $^{2 *}$, IGNACE VERGOTE $^{3}$, \\ ADRIAAN VANDERSTICHELE ${ }^{3}$, JALID SEHOULI $^{4}$, ELENA BRAICU $^{4}$, SVEN MAHNER $^{5}$, \\ DAN CACSIRE CASTILLO-TONG $^{6}$, ROBERT ZEILLINGER ${ }^{7}$ and THERESIA THALHAMMER ${ }^{1}$
}

\begin{abstract}
${ }^{1}$ Department of Pathophysiology and Allergy Research, Center for Pathophysiology, Infectiology and Immunology, Medical University of Vienna; ${ }^{2}$ Department of Gynaecology and Gynaecological Oncology, Comprehensive Cancer Center, Medical University of Vienna, A-1090 Vienna, Austria; ${ }^{3}$ Division of Gynaecological Oncology, Department of Obstetrics and Gynaecology, Catholic University of Leuven, University Hospital, B-3000 Leuven, Belgium; ${ }^{4}$ Department of Gynaecology, European Competence Center for Ovarian Cancer, Virchow Clinic Campus, Medical University of Berlin, D-13353 Berlin; ${ }^{5}$ Department of Gynaecology and Obstetrics, University of Munich, D-80539 Munich, Germany;

${ }^{6}$ Translational Gynaecology Group, Department of Obstetrics and Gynaecology, Comprehensive Cancer Center, Medical University of Vienna; ${ }^{7}$ Molecular Oncology Group, Department of Obstetrics and Gynaecology, Comprehensive Cancer Center, Medical University of Vienna, A-1090 Vienna, Austria
\end{abstract}

Received September 13, 2016; Accepted January 13, 2017

DOI: $10.3892 / 01.2017 .5969$

\begin{abstract}
E2) can contribute to the progression of epithelial ovarian cancer (EOC). Although the majority of patients with EOC are postmenopausal woman, when de novo estrogen production in the ovary has ceased, ovarian cancer cells remain exposed to estrogens synthesized locally in the cancer cells from inactive sulfonated steroid hormone precursors-such as estrone sulfate taken up from the circulation via the sulfatase pathway. An abundance of the estrogen-modifying enzymes, including estrogen-activating steroid sulfatase (STS) and estrogen-inactivating estrogen-sulfotransferase (SULT1E1), is important for providing active estrogen to EOC cells. Therefore, the present study determined the levels of SULT1E1, STS and estrogen receptor $\alpha(\mathrm{ER} \alpha)$ protein in paraffin-embedded specimens from 206 patients with Federation of Gynecology and Obstetrics stage II-IV EOC treated with debulking surgery and standard platinum-based adjuvant chemotherapy. The levels of STS, SULT1E1 and ER $\alpha$ were assessed by automated quantitative microscopy-based
\end{abstract}

Correspondence to: Dr Theresia Thalhammer, Department of Pathophysiology and Allergy Research, Center for Pathophysiology, Infectiology and Immunology, Medical University of Vienna, 3Q Wahringer Guertel 18-20, A-1090 Vienna, Austria

E-mail: theresia.thalhammer@meduniwien.ac.at

*Contributed equally

Key words: epithelial ovarian cancer, estrogen, estrogen metabolizing enzymes, STS, sulfotransferase, SULT1E1, E2, ovarian cancer image analysis subsequent to immunohistochemical staining. Significantly higher SULT1E1 levels were observed in better differentiated EOC tumors compared to grade 3 EOC tumors $(\mathrm{P}=0.001)$. STS and SULT1E1 levels were positively associated with $E R \alpha$ abundance $(\mathrm{P}<0.001$ and $\mathrm{P}=0.001$, respectively). In advanced stage high-grade serous EOC (HGSOC; $n=132$ ), the most frequent and lethal type of ovarian cancer, SULT1E1 expression was significantly associated with a better overall survival rate (hazard ratio $0.66,95 \%$ confidence interval, $0.45-0.94 ; \mathrm{P}=0.005)$. These results highlight the importance of SULT1E1-mediated estrogen inactivation in EOC, particularly HGSOC. Therefore, targeting the sulfatase pathway is a potential endocrine therapeutic intervention for certain patients with estrogen-responsive EOC.

\section{Introduction}

Epithelial ovarian cancer (EOC) remains the most lethal type of gynecologic malignancy in postmenopausal women in industrialized countries (1). Comprising a heterogeneous group of malignant tumors, EOC presents with distinct clinicopathological and biological characteristics. The majority $(\sim 75 \%)$ of all incidences of ovarian cancer are classified as high-grade serous epithelial ovarian cancer (HGSOC), which is hypothesized to originate from serous tubal intraepithelial lesions in the fallopian tubes rather than the ovarian epithelium (2). Less frequently observed are endometrial and clear cell carcinomas, associated with endometrial cells, while mucinous carcinomas are associated with gastrointestinal tract tissue. Molecular changes in the genes encoding tumor protein p53, breast cancer type 1 susceptibility protein (BRCA1), BRCA2, GTPase Kras and proto-oncogene B-Raf are known to be fundamental for the development of different subtypes of EOC. The results of epidemiological studies have also revealed 
that reproductive factors, which determine the exposure of females to steroid hormones, particularly estrogens, serve a role in the development of all subtypes of EOC, although their contribution varies between subtypes (3-5). While the application of hormonal contraceptives and a higher number of pregnancies reduce the risk for all ovarian cancer types, estrogen-only hormone replacement therapy (HRT) was found to increase the risk for serous and endometrioid EOCs. A higher overall lifetime exposure to estrogens through an early menarche and late menopause was demonstrated to be associated with an increased risk of non-serous ovarian cancers but it may not increase the incidence of HGSOC (6-10).

When investigating the effect of estrogens on EOC cells, it is of note that these tumors occur predominately in postmenopausal women, at a time when the de novo synthesis of the most active form of estrogen, $17 \beta$-estradiol (E2), in the ovary has ceased. However, cells in the ovary and other estrogen-sensitive tissues, such as the endometrium and breast, remain exposed to E2 provided from circulating estrogen precursors, particularly estrone sulfate (E1S), taken up from the circulation via specific transport proteins, including members of the organic anion transporting polypeptide family (Fig. 1) (11). A study by Sasano et al (12) demonstrated that in postmenopausal women, the local concentration of estrogen in breast and endometrial carcinoma are of a similar level compared with those in premenopausal women. This local estrogen production has been demonstrated to be important for the progression of hormone-dependent cancer of the breast and endometrium (13-15).

In breast cancer cells, the local production of E2 from E1S via the sulfatase pathway has been revealed to exceed the production of E2 from androgenic precursors, including dehydroepiandrosterone sulfate (DHEA-S) via the aromatase pathway (16). In the sulfatase pathway, subsequent to the cellular uptake of E1S, which is the most abundant type of estrogen in the circulation of postmenopausal women (17), estrone (E1) that has weak estrogenic activity is formed by the enzymatic activity of the steroid sulfatase (STS; Fig. 1). Subsequently, E2 is produced from E1 by the activity of $17 \beta$-oxoreductase. Conversely, E2 may be reverted back to E1 via the oxidative function of $17 \beta$-hydroxysteroid dehydrogenase. As an inactivation pathway, E2 conjugation with sulfonate by estrogen sulfotransferase (SULT1E1) and phenol sulfotransferase (SULT1A1) produces estradiol sulfate (E2S), which exhibits minimal estrogenic activity. Conversely, STS may produce active estrogens from E2S and E1S, suggesting that an increase in the level of STS raises the levels of active estrogens and contributes to the progression of estrogen-sensitive types of cancer. Increased expression of STS has previously been identified to be associated with breast cancer progression (18). Similarly, in a small group of patients with advanced stage ovarian cancer an increased level of STS activity was revealed to be associated with a worse progression-free survival (PFS) (19). Additionally, STS mRNA was detectable in ovarian cancer cells, with similar levels detected in tissue from pre- and postmenopausal women (20), but was not detectable in normal ovarian surface epithelial cells (14).

Exerting the opposite effect to STS, the increased expression of estrogen-inactivating sulfotransferases may have beneficial effects in malignant and non-malignant diseases known to be sensitive to estrogens. For example, in ovarian endometriosis the expression of SULT1E1, but not of SULT1A1, was demonstrated to be decreased compared with normal tissue (20), suggesting that SULT1E1 is important for estrogen inactivation. The importance of SULT1E1 expression was also revealed in breast cancer, where increased levels of the enzyme are associated with a decreased risk of recurrence and an improved prognosis (12). Similarly, the overexpression of SULT1E1 was identified to reduce the growth of hormone-sensitive breast cancer cells and block tumorigenesis in a xenograft cancer model (21). In endometrial cancer, higher levels of STS compared with SULT1E1 were correlated with a poorer prognosis (22). For ovarian cancer, no studies regarding SULT1E1 are available at present, to the best of our knowledge.

The effects of estrogen on cell differentiation and proliferation are largely mediated via the binding and activation of nuclear estrogen receptor (ER) $\alpha$ and ER $\beta$. Numerous studies have revealed that in estrogen-sensitive tumors, including breast and endometrium carcinoma, ERs and estrogen activation via the sulfatase pathway serve a crucial role in tumor progression $(23,24)$. Similarly, in preclinical models of ovarian cancer, a high expression of $\mathrm{ER} \alpha$ was identified to promote tumor progression and the development of metastasis by inducing epithelial-mesenchymal transition. In ovarian cancer patients, ER $\alpha$ was demonstrated to be important for tumor progression and a potential target for endocrine therapy of EOC $(25,26)$. However, the success of endocrine therapy targeting ER $\alpha$ remains limited, although the vast majority of EOC subtypes, particularly HGSOC and endometrioid tumors, were found to express the receptor (27). Therefore, hormonal treatment selection based on ER, SULT1E1 and STS expression may lead to an improved patient outcome.

The present study aimed to examine the expression of STS, SULT1E1 and ER $\alpha$ protein in a well-defined cohort of patients with advanced EOC. Additionally, the prognostic value of these targets was determined in patients with HGSOC histology, being the most frequently observed and lethal subtype of ovarian cancer.

\section{Materials and methods}

Patients. The levels of STS, SULT1E1 and ER $\alpha$ protein were examined in paraffin-embedded tumor sections from 206 patients with EOC. Samples were collected as part of the European Union-funded specific targeted research project 'Ovarian Cancer: Diagnosis of a silent killer'. Patients with FIGO stage II-IV ovarian cancer were included from December 2005 to November 2008 in The Department of Gynecology at Charité, Medical University Berlin, Germany; The Department of Obstetrics and Gynecology and Gynecologic Oncology, University Hospital Leuven, Belgium: Department of Gynecology, University Medical Center Hamburg-Eppendorf, Hamburg, Germany; The Department of Obstetrics and Gynecology, Medical University of Vienna, Austria. Patients with other malignancies were excluded. Informed consents were obtained from all patients. The study protocol was approved by the Ethics Committees of the participating institutions (approval nos. EK207/2003: Berlin; ML2524: Leuven; HEK190504: Hamburg; EK366 and EK260: Vienna), with the 


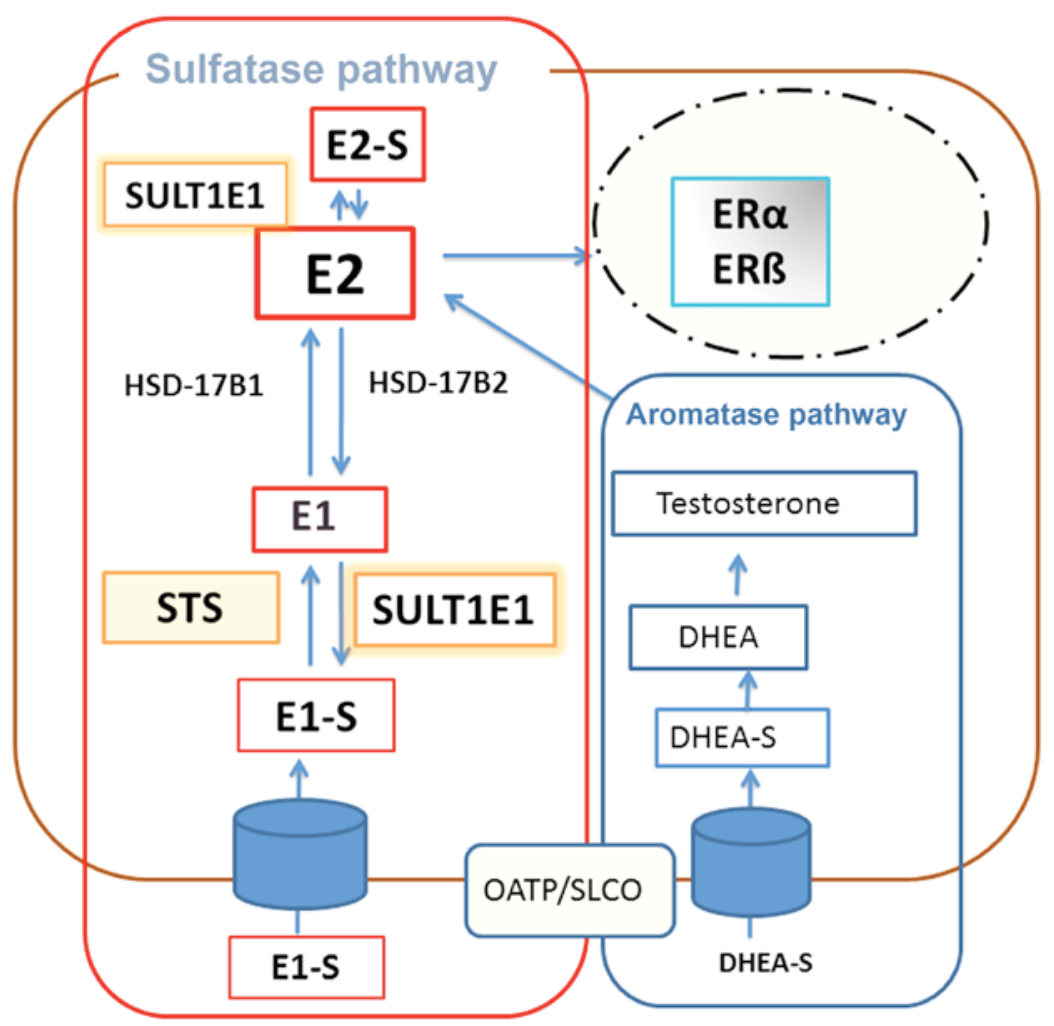

Figure 1. Intratumoral synthesis of E2 and its inactivation by sulfate conjugation from circulating steroid hormone precursors in cancer cells. E2 is synthesized from E1S in the sulfatase pathway, and from DHEA-S in the aromatase pathway. E1S and DHEA-S are taken up from the circulation by transporters, such as those from the OATP family encoded by SCLO. In the sulfatase pathway, HSD-17ß1 generates E2 from E1S, while E1 and E2 are inactivated by sulfonation by SULT1E1. Sulfonated estrogens do not bind to the ER $\alpha$ or ER $\beta$. Aromatase generates E2 from androgenic precursors via the aromatase pathway. E2, 17ß-estradiol; -S, sulfate; E1, estrone; STS, steroid sulfatase; SULT1E1, estrogen sulfotransferase; ER, estrogen receptor; DHEA, dehydroepiandrosterone sulfate; HSD-17 $\beta$, 17ß-hydroxysteroid dehydrogenase; OATP, organic anion transporting polypeptides; SLCO, solute carrier for organic anions.

permission to characterize new molecular prognostic factors for patients with advanced EOC.

Only patients with Federation of Gynecology and Obstetrics (FIGO) stage II-IV (27) EOC receiving standard treatment, debulking surgery and platinum-based chemotherapy with taxol derivatives were included in the present study. Patients presenting with benign ovarian diseases, low malignant potential ovarian cancer, FIGO stage I EOC and secondary malignant diseases were excluded. All patients provided preoperative written informed consent prior to enrollment in the present study. Histopathological grading was done for all tumors, grade 1 -grade 3 , according to tissue differentiation (28).

PFS was defined as the time interval between the date of primary surgery and the date of first progression or recurrence of cancer. Disease progression subsequent to first-line chemotherapy was diagnosed by clinical examination, tumor imaging or by a $>2$-fold increase in the nadir serum cancer antigen-125 level. Overall survival (OS) was defined as the time interval between initial cytoreductive surgery, EOC-associated mortality or last follow-up. Clinical and histopathological evaluation was performed under the supervision of experienced gynecologic oncologists and pathologists in the participating institutions of the present study.

Immunohistochemical (IHC) staining. IHC staining was performed on tissue microarrays (TMAs) containing 2 core sections/tumor tissue (1 $\mathrm{mm}$ diameter). Subsequent to heating the sections in a tissue-drying oven for $1 \mathrm{~h}$ at $60^{\circ} \mathrm{C}$, antigen retrieval was performed for 10 min with DEPP-buffer $(\mathrm{pH} 9.0$; Eubio, Vienna, Austria) in a microwave. Blocking for endogenous peroxidase and applying the Lab Vision ${ }^{\mathrm{TM}}$ Ultra V Block (UltraVision LP Detection System; Thermo Fisher Scientific, Inc., Waltham, MA, USA). The UltraVision LP Detection System was used according to the manufacturer's instructions. Incubation with antibodies directed against STS (cat. no. N1C3; dilution, 1:400; GeneTex, Inc., Irvine, CA, USA) and SULT1E1 (cat. no. 12522-1-AP; dilution, 1:200; ProteinTech Group, Inc., Chicago, IL, USA) was performed overnight at $4^{\circ} \mathrm{C}$. Antigen retrieval and staining for ER $\alpha$ using mouse IgG1 $\kappa$ directed against recombinant ER $\alpha$ protein (clone 1D5; cat. no. MA5-13191; Thermo Fisher Scientific, Inc.) was performed as previously described by Aust et al (29). Sections were treated with HRP Polymer prior to the application of a DAB Plus Chromogen/DAB Plus Substrate mixture (ratio, 1:40) (both UltraVision LP Detection System; Thermo Fisher Scientific, Inc.). The nuclei were counterstained with hematoxylin and the slides were then embedded in Fluoromount (SouthernBiotech, Birmingham, AL, US).

Evaluation of IHC staining results. Images of the TMAs were acquired with the automated quantitative microscopy-based image analysis system TissueFAXS PLUS (TissueGnostics $\mathrm{GmbH}$, Vienna, Austria) using the 20X objective. A minimum of 3 regions of interest were manually drawn in the tumor areas based on the individual tissue structures and data were acquired 


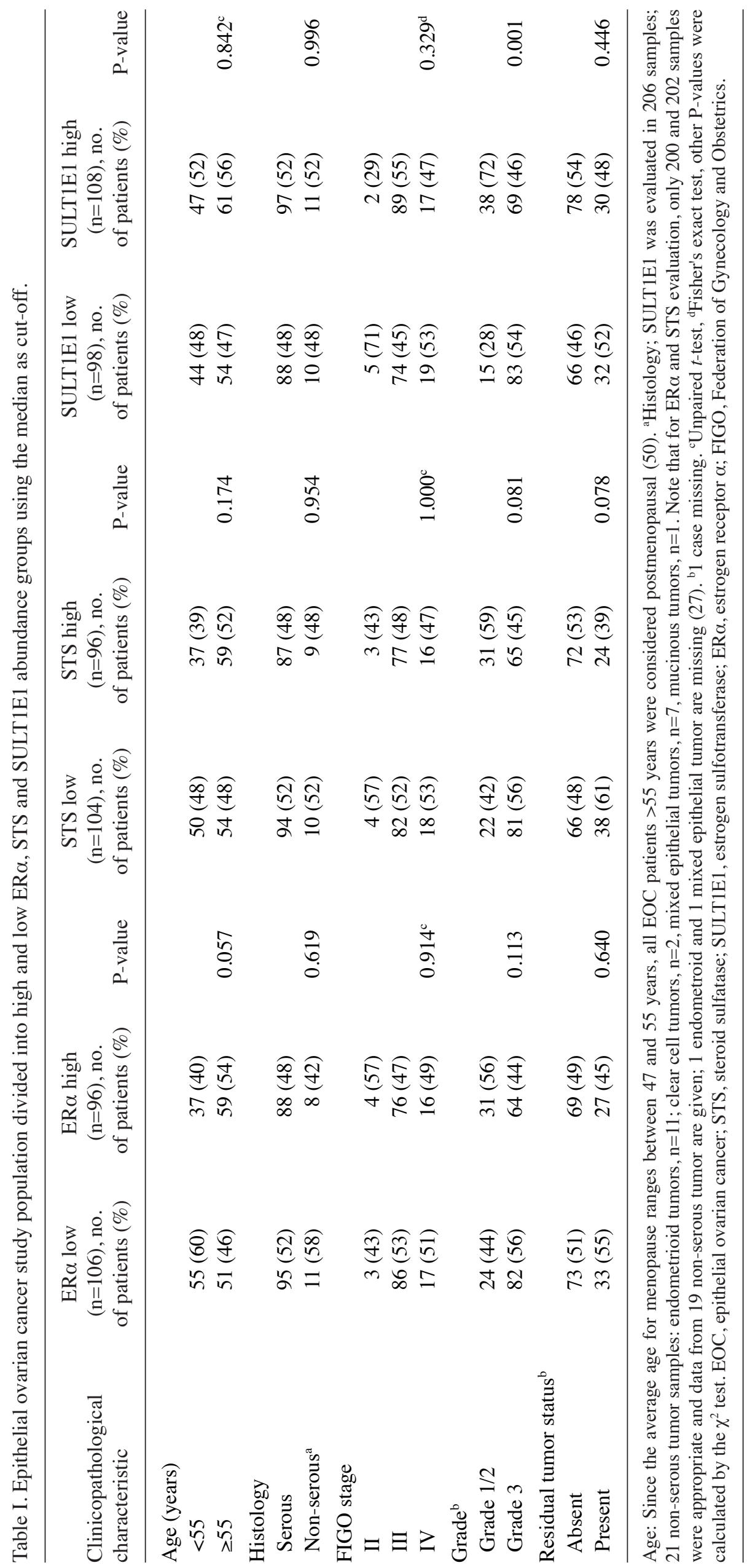



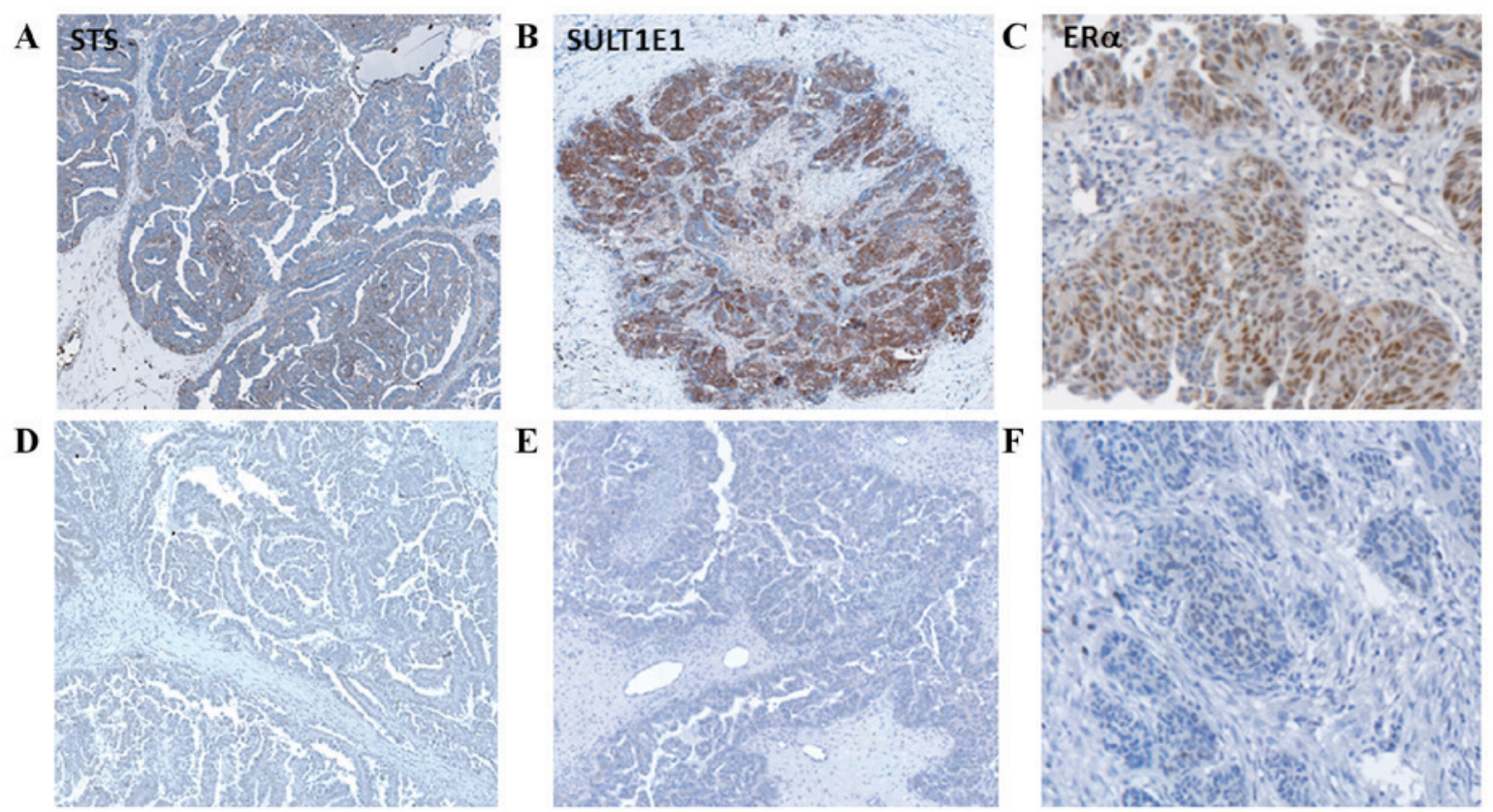

Figure 2. Representative images of immunohistochemical staining for (A) STS, (B) SULT1E1 and (C) ER $\alpha$ subsequent in paraffin-embedded sections from serous epithelial ovarian cancer. Cytoplasmic staining was observed for STS and SULT1E1, while ER $\alpha$ staining was predominantly observed in the nucleus. Respective images from negative control samples for (D) STS, (E) SULT1E1 and (F) ER $\alpha$. Magnification, x200. STS, steroid sulfatase; SULT1E1, estrogen sulfotransferase; ER $\alpha$, estrogen receptor $\alpha$.

for these regions. HistoQuest software (version 3.0.3.0161; TissueGnostics $\mathrm{GmbH}$ ) was used to determine the staining intensity as gray values of between 0 and 250 arbitrary units. Gray values were determined from the minimum (set to 0 ) to maximum (set to 250) of the average optical density of the DAB stained target using the HistoQuest software. In the histochemical analysis color shades are picked manually in an automated color separation produces to give a gray value channel image for each marker (30).

The proportional expression, defined as the percentage of positively stained tumor cells, was used for the calculation of the, mean of mean intensity as described previously (31). Staining intensity in the cytoplasm (STS and SULT1E1) or in the nucleus $(E R \alpha)$ was assessed subsequent to setting a cut-off associated with values from negative control images generated by the application of non-immunogenic IgG or phosphate-buffered saline instead of the first antibody. To correct for false positive events, a specific gate according to cell size and intensity staining was defined and applied homogenously to all analyzed samples. For the assessment of data, only samples with a sufficient tissue quality, including structural integrity of the tissue and clear staining quality with an appropriate negative control, were chosen for quantitative microscopic analysis. All procedures were guided, monitored and approved by a pathologist.

Statistical analysis. Statistical analysis was performed using SPSS software (version 19.0; IBM SPSS, Armonk, NY, USA). Descriptive statistics such as the mean, median, frequency and percentage were used to summarize the data. Data was log-transformed to reach a normal distribution, as appropriate. The abundance values of STS, SULT1E1 and ER $\alpha$ were grouped into high and low abundance groups using the median as cut-off. Statistical correlations between low and high abundance groups and clinicopathological parameters were assessed by the $\chi^{2}$ test, Fisher's exact test and unpaired $t$-tests, as appropriate. To assess the impact of STS, SULT1E1 and ER $\alpha$ on PFS and OS, linear log-transformed values were used in univariate and multivariate Cox regression analyses. Multivariate analyses were performed using stepwise regression (backward elimination). $\mathrm{P}<0.05$ was considered to indicate a statistically significant difference.

\section{Results}

EOC tumors. The clinicopathological characteristics of the 206 patients with EOC are illustrated in Table I. The median observation period was 68 months (range, 1-96 months). The median age of patients at the time of cytoreductive surgery was 56 years (range, 26-85 years), whereby the macroscopic cytoreduction rate was $70 \%$. Additional clinicopathological characteristics of this cohort have previously been described (32).

Representative images of STS, SULT1E1 and ER $\alpha$ staining compared with the negative controls are illustrated in Fig. 2. Cytoplasmic staining in the tumor cells was visible for STS and SULT1E1. Staining of cells in the tumor stroma was weak and infrequent in immune cells. Immunoreactive ER $\alpha$ staining was observed in the tumor cell nuclei and, occasionally, in the perinuclear region.

For the evaluation of the abundance of STS, SULT1E1 and ER $\alpha$, only tissue samples fulfilling the requirements for a quantitative microscopy-based image analysis, aforementioned in the methods section, were included in the present study. The mean levels of immunoreactive STS, SULT1E1 and ER $\alpha$ were calculated and patients were stratified into high and low target abundance groups (Table I). Finally, tumor tissue samples from 206, 200 and 202 patients were evaluated for SULT1E1, STS 
and ER $\alpha$ abundance, respectively. No significant differences regarding clinicopathological characteristics (tumor histology, age, FIGO stage and residual tumor) were observed between the low and high ER $\alpha$ and STS abundance groups. However, the degree of tissue differentiation (28) was important for the expression level as a significantly higher SULT1E1 abundance was observed in more differentiated grade $1 / 2$ tumors compared with less differentiated grade 3 tumors $(\mathrm{P}=0.001)$. A total of $59 \%$ of the collective of grade $1 / 2$ compared with $45 \%$ grade 3 tumors exhibited high STS abundance $(\mathrm{P}=0.081)$. In addition, STS and SULT1E1 protein levels were positively correlated with each other $(\mathrm{P}<0.001)$ and with $\mathrm{ER} \alpha(\mathrm{P}<0.001$ and $\mathrm{P}=0.001$, respectively; data not shown).

HGSOC. Different histological subtypes of EOC have distinctive characteristics regarding the origin of the tumor, the growth pattern, the sensitivity to chemotherapy and the prognosis. As it is recommended to perform survival analyses for different ovarian cancer subtypes separately (33), a homogenous group of patients with a long observation period were used for survival analyses. The data of 137 patients with HGSOC were used to assess the impact of SULT1E1, STS, and ER $\alpha$ abundance on clinical outcome (Table II). During the median observation period of 68 months (range, 1-96 months), a total of $84(64 \%)$ patients succumbed to the disease and $113(86 \%)$ patients experienced a tumor recurrence (data not shown).

Multivariate Cox regression analysis revealed that SULT1E1 abundance was a significant independent predictor for OS (hazard ratio, 0.66; 95\% confidence interval 0.45-0.94; $\mathrm{P}=0.005$; Table II). Notably, neither ER $\alpha$ nor STS abundance exhibited a significant impact on OS. These results suggest that patients with higher SULT1E1 levels have a better prognosis, independent from the most relevant confounding parameters demonstrated in Table II. The abundance of ER $\alpha$, STS and SULT1E1 were not significantly associated with PFS (Table II).

Non-serous tumors. STS, SULT1E1 and ER $\alpha$ were also detected in non-serous tumors (Table I). Due to the limited number of patients ( $\mathrm{n}=21$; endometrioid tumors, $\mathrm{n}=11$; clear cell tumors, $\mathrm{n}=2$; mixed epithelial tumors, $\mathrm{n}=7$; mucinous tumors, $\mathrm{n}=1$ ) and the heterogeneity of the tumors, distinct survival analyses were not performed for the non-serous tumor group.

\section{Discussion}

The intratumoral production of E2 from circulating E1S via the sulfatase pathway, including the enzymes STS for estrogen activation and SULT1E1 for estrogen inactivation, serves an important role in estrogen-associated cancer of the breast and endometrium (34). Therefore, the present study determined the levels of STS, SULT1E1 and ER $\alpha$ in a well-defined cohort of 205 EOC patients. This revealed that SULT1E1 abundance exhibited a significant independent prognostic value for OS in 137 patients with HGSOC treated with debulking surgery and standard platinum-based adjuvant chemotherapy. A high level of SULT1E1 was associated with a longer OS in patients with HGSOC, which is concordant with results from previous studies of breast and endometrial cancer. In these tumors, high SULT1E1 levels were found to be associated with a better prognosis for patients, as demonstrated by a decreased risk of 
recurrence and an increased OS $(13,22)$. This beneficial role of SULT1E1 is further supported by the finding that SULT1E1 is typically downregulated in malignant tissue $(34,35)$. In addition, preclinical studies have demonstrated that the inactivation of E2 by sulfonation reduces the proliferative effects of estrogens on hormone-sensitive tumor cells (36). Furthermore, the conjugation of estrogens by SULT1E1 creates water-soluble estrogen sulfates, which may be rapidly excreted from the cells. Effective sulfonation and rapid excretion of the sulfonated E2 may prevent the metabolic activation of the hormone into potential mutagenic catechol metabolites, which increase the mutation rate of DNA and lead to chromosomal instability $(37,38)$. Since the present study did not identify a significant association between STS and OS/PFS, in HGSOC SULT1E1 may be of a higher relevance compared with STS for estrogen inactivation and estrogen homeostasis. However, Chura et al (19) reported that a decreased level of STS activity was associated with a higher OS, based on a smaller cohort of patients with ovarian cancer $(n=37)$ with no additional characterization of the tumor subtypes. Therefore, the clinicopathological characteristics and number of patients included in the present study may explain the diverging data in regards to STS, particularly as a large number of patients with HGSOC were included. However, data on the association between STS expression and tumor progression remain controversial even in otherwise well-studied endometrial carcinomas. This may be partly explained by different approaches to investigating estrogen-modifying enzymes. For example, Abulafia et al (39) identified that there was higher STS activity in endometrial carcinoma compared with normal endometrial tissues using DHEA-sulfate as a substrate, but Tanaka et al (40) reported a decreased level of STS activity in endometrial carcinoma compared with normal endometrium as the lining of the uterus and endometrial adenocarcinoma-derived cells using E1S as a substrate.

Despite the uncertainty with respect to the role of STS in the progression of gynecological cancers and the limited data from clinical trials, STS has been proposed to be a potential hormonal therapeutic target for the treatment of estrogen-associated cancer (41). It must be considered that the regulation of STS expression is subject to various feedback mechanisms, including the levels of active estrogens (42). Therefore, in EOC the increased inactivation of E2 by low levels of SULT1E1 may cause an upregulation of STS. Indeed, a significant association between the expression of SULT1E1/STS and ER $\alpha$ was identified in the present study, similarly to the results of a previous study investigating breast cancer (43). In the present study, although STS and SULT1E1 abundance was significantly associated and the abundance of these enzymes was significantly associated with the level of $E R \alpha$, the receptor itself exhibited no significant impact on PFS or OS. In a study with a large cohort of patients with serous EOC, the expression levels of ER $\alpha$ and ER $\beta$ did not provide any prognostic information $(14,44)$.

From the data obtained, the present study concludes that targeting estrogen-modifying enzymes in the sulfatation pathway is a potential strategy for the endocrine therapy of estrogen-sensitive ovarian cancer. Targeting the sulfatase pathway was previously suggested as an endocrine therapy option for breast cancer (45). In a mouse xenograft model, blocking aromatase together with the application of the STS inhibitor STX64 reduced the estrogen level in tumors (46). This is important as the application of the estrogen-depleting aromatase inhibitor exemestane can cause an upregulation of intratumoral STS, leading to therapy resistance (47). Whether SULT1E1 may also be downregulated through feedback mechanisms remains unknown. However, STX64 was found to inhibit the growth of the ER ${ }^{+}$ovarian carcinoma OVCAR-3 cell line and thus may be considered a target for ovarian cancer therapy (48). The importance of SULT1E1 was also identified in the use of the prodrug tibolone, which improves bone structure in postmenopausal women as it is metabolized into 2 products with antagonistic effects on ER $\alpha$. In the endometrium, SULT1E1 effectively converts these 2 metabolic products to inactive sulfate conjugates, preventing ER $\alpha$ activation and reducing the risk of endometrial cancer induction (49).

In conclusion, the results of the present study suggest that estrogen-modifying enzymes in the sulfatase pathway, such as STS and SULT1E1, are potential endocrine therapy targets for the treatment of patients with EOC.

\section{Acknowledgements}

The present study was supported by the European Union-funded Sixth Framework Programme Specific Targeted Research Project Ovarian Cancer: Diagnosis of a silent killer (grant no. 018698).

\section{References}

1. American Cancer Society, American Cancer Society 2016: Ovarian epithelial, fallopian tube and primary peritoneal cancer treatment $\left(\mathrm{PDQ}^{\circledR}\right)$. https://www.cancer.gov/types/ovarian/hp/ ovarian-epithelial-treatment-pdq. Accessed January 17, 2017.

2. Kessler M, Fotopoulou C and Meyer T: The molecular fingerprint of high grade serous ovarian cancer reflects its fallopian tube origin. Int J Mol Sci 14: 6571-6596, 2013.

3. Brown J and Frumovitz M: Mucinous tumors of the ovary: Current thoughts on diagnosis and management. Curr Oncol Rep 16: 389, 2014.

4. Sapiezynski J, Taratula O, Rodriguez-Rodriguez L and Minko T: Precision targeted therapy of ovarian cancer. J Control Release 243: 250-268, 2016.

5. Chuffa LG, Lupi-Junior LA, Costa AB, A morim JP and Seiva FR: The role of sex hormones and steroid receptors on female reproductive cancers. Steroids 118: 93-108, 2017.

6. Beral V; Million Women Study Collaborators, Bull D, Green J and Reeves G: Ovarian cancer and hormone replacement therapy in the Million Women Study. Lancet 369: 1703-1710, 2007.

7. Kurian AW, Balise RR, McGuire V and Whittemore AS: Histologic types of epithelial ovarian cancer: Have they different risk factors? Gynecol Oncol 96: 520-530, 2005.

8. Rice LW: Hormone prevention strategies for breast, endometrial and ovarian cancers. Gynecol Oncol 118: 202-207, 2010.

9. Schock H, Surcel HM, Zeleniuch-Jacquotte A, Grankvist K, Lakso HA, Fortner RT, Kaaks R, Pukkala E, Lehtinen M, Toniolo P and Lundin E: Early pregnancy sex steroids and maternal risk of epithelial ovarian cancer. Endocr Relat Cancer 21: 831-844, 2014.

10. Trabert B, Brinton LA, Anderson GL, Pfeiffer RM, Falk RT, Strickler HD, Sliesoraitis S, Kuller LH, Gass ML, Fuhrman BJ, et al: Circulating estrogens and postmenopausal ovarian cancer risk in the Women's Health initiative observational study. Cancer Epidemiol Biomarkers Prev 25: 648-656, 2016.

11. Svoboda M, Wlcek K, Taferner B, Hering S, Stieger B, Tong D, Zeillinger R, Thalhammer T and Jäger W: Expression of organic anion-transporting polypeptides $1 \mathrm{~B} 1$ and 1B3 in ovarian cancer cells: Relevance for paclitaxel transport. Biomed Pharmacother 65: 417-426, 2011. 
12. Sasano H, Miki Y, Nagasaki S and Suzuki T: In situ estrogen production and its regulation in human breast carcinoma: From endocrinology to intracrinology. Pathol Int 59: 777-789, 2009.

13. Suzuki T, Miki Y, Nakamura Y, Ito K and Sasano H: Steroid sulfatase and estrogen sulfotransferase in human carcinomas. Mol Cell Endocrinol 340: 148-153, 2011.

14. Ren X, Wu X, Hillier SG, Fegan KS, Critchley HO, Mason JI, Sarvi S and Harlow CR: Local estrogen metabolism in epithelial ovarian cancer suggests novel targets for therapy. J Steroid Biochem Mol Biol 150: 54-63, 2015.

15. Rizner TL: Estrogen biosynthesis, phase I and phase II metabolism, and action in endometrial cancer. Mol Cell Endocrinol 381: 124-139, 2013.

16. McNamara KM and Sasano H: The intracrinology of breast cancer. J Steroid Biochem Mol Biol 145: 172-178, 2015.

17. Müller P, Rothschild SI, Arnold W, Hirschmann P, Horvath L, Bubendorf L, Savic S and Zippelius A: Metastatic spread in patients with non-small cell lung cancer is associated with a reduced density of tumor-infiltrating $\mathrm{T}$ cells. Cancer Immunol Immunother 65: 1-11, 2016.

18. Hanamura T, Niwa T, Gohno T, Kurosumi M, Takei $H$, Yamaguchi Y, Ito $\mathrm{K}$ and Hayashi S: Possible role of the aromatase-independent steroid metabolism pathways in hormone responsive primary breast cancers. Breast Cancer Res Treat 143 : 69-80, 2014.

19. Chura JC, Blomquist $\mathrm{CH}$, Ryu HS and Argenta PA: Estrone sulfatase activity in patients with advanced ovarian cancer. Gynecol Oncol 112: 205-209, 2009.

20. Hevir N, Ribič-Pucelj M and Lanišnik Rižner T: Disturbed balance between phase I and II metabolizing enzymes in ovarian endometriosis: A source of excessive hydroxy-estrogens and ROS? Mol Cell Endocrinol 367: 74-84, 2013.

21. Xu Y, Liu X, Guo F, Ning Y, Zhi X, Wang X, Chen S, Yin L and Li X: Effect of estrogen sulfation by SULT1E1 and PAPSS on the development of estrogen-dependent cancers. Cancer Sci 103 : 1000-1009, 2012.

22. Utsunomiya H, Ito K, Suzuki T, Kitamura T, Kaneko C, Nakata T, Niikura H, Okamura K, Yaegashi N and Sasano H: Steroid sulfatase and estrogen sulfotransferase in human endometrial carcinoma. Clin Cancer Res 10: 5850-5856, 2004.

23. Purohit A, Woo LW and Potter BV: Steroid sulfatase: A pivotal player in estrogen synthesis and metabolism. Mol Cell Endocrinol 340: 154-160, 2011.

24. Mungenast $\mathrm{F}$ and Thalhammer T: Estrogen biosynthesis and action in ovarian cancer. Front Endocrinol (Lausanne) 5: 192 2014.

25. Park SH, Cheung LW, Wong AS and Leung PC: Estrogen regulates Snail and Slug in the down-regulation of E-cadherin and induces metastatic potential of ovarian cancer cells through estrogen receptor alpha. Mol Endocrinol 22: 2085-2098, 2008.

26. Haring J, Schuler S, Lattrich C, Ortmann O and Treeck O: Role of estrogen receptor $\beta$ in gynecological cancer. Gynecol Oncol 127 673-676, 2012.

27. Voutsadakis IA: Hormone receptors in serous ovarian carcinoma: Prognosis, pathogenesis, and treatment considerations. Clin Med Insights Oncol 10: 17-25, 2016.

28. Silverberg SG: Histopathologic grading of ovarian carcinoma: A review and proposal. Int J Gynecol Pathol 19: 7-15, 2000.

29. Aust S, Bachmayr-Heyda A, Pateisky P, Tong D, Darb-Esfahani S, Denkert C, Chekerov R, Sehouli J, Mahner S, Van Gorp T, et al: Role of TRAP1 and estrogen receptor alpha in patients with ovarian cancer-a study of the OVCAD consortium. Mol Cancer 11: 69, 2012

30. Kounnis V, Ioachim E, Svoboda M, Tzakos A, Sainis I, Thalhammer T, Steiner G and Briasoulis E: Expression of organic anion-transporting polypeptides $1 \mathrm{~B} 3,1 \mathrm{~B} 1$, and $1 \mathrm{~A} 2$ in human pancreatic cancer reveals a new class of potential therapeutic targets. Onco Targets Ther 4: 27-32, 2011

31. Remmele W and Stegner HE: Recommendation for uniform definition of an immunoreactive score (IRS) for immunohistochemical estrogen receptor detection (ER-ICA) in breast cancer tissue. Pathologe 8: 138-140, 1987 (In German).

32. Chekerov R, Braicu I, Castillo-Tong DC, Richter R, Cadron I, Mahner S, Woelber L, Marth C, Van Gorp T, Speiser P, et al: Outcome and clinical management of 275 patients with advanced ovarian cancer International Federation of Obstetrics and Gynecology II to IV inside the European Ovarian Cancer Translational Research Consortium-OVCAD. Int J Gynecol Cancer 23: 268-275, 2013.
33. Kobel M, Kalloger SE, Boyd N, McKinney S, Mehl E, Palmer C, Leung S, Bowen NJ, Ionescu DN, Rajput A, et al: Ovarian carcinoma subtypes are different diseases: Implications for biomarker studies. PLoS Med 5: e232, 2008.

34. Rizner TL: The important roles of steroid sulfatase and sulfotransferases in gynecological diseases. Front Pharmacol 7: 30, 2016.

35. Smuc T and Rizner TL: Aberrant pre-receptor regulation of estrogen and progesterone action in endometrial cancer. Mol Cell Endocrinol 301: 74-82, 2009.

36. Li L, He D, Wilborn TW, Falany JL and Falany CN: Increased SULT1E1 activity in HepG2 hepatocytes decreases growth hormone stimulation of STAT5b phosphorylation. Steroids 74 : 20-29, 2009.

37. Falany CN, Comer KA, Dooley TP and Glatt H: Human dehydroepiandrosterone sulfotransferase. Purification, molecular cloning, and characterization. Ann N Y Acad Sci 774: 59-72, 1995.

38. Adjei AA and Weinshilboum RM: Catecholestrogen sulfation: Possible role in carcinogenesis. Biochem Biophys Res Commun 292: 402-408, 2002.

39. Abulafia O, Lee YC, Wagreich A, Economos K, Serur E and Nacharaju VL: Sulfatase activity in normal and neoplastic endometrium. Gynecol Obstet Invest 67: 57-60, 2009.

40. Tanaka K, Kubushiro K, Iwamori Y, Okairi Y, Kiguchi K, Ishiwata I, Tsukazaki K, Nozawa S and Iwamori M: Estrogen sulfotransferase and sulfatase: Roles in the regulation of estrogen activity in human uterine endometrial carcinomas. Cancer Sci 94: 871-876, 2003.

41. Purohit A, Fusi L, Brosens J, Woo LW, Potter BV and Reed MJ: Inhibition of steroid sulphatase activity in endometriotic implants by 667 COUMATE: A potential new therapy. Hum Reprod 23: 290-297, 2008

42. Reed MJ, Purohit A, Woo LW, Newman SP and Potter BV: Steroid sulfatase: Molecular biology, regulation, and inhibition. Endocr Rev 26: 171-202, 2005.

43. Zaichuk T, Ivancic D, Scholtens D, Schiller C and Khan SA: Tissue-specific transcripts of human steroid sulfatase are under control of estrogen signaling pathways in breast carcinoma. J Steroid Biochem Mol Biol 105: 76-84, 2007.

44. Jonsson JM, Skovbjerg Arildsen N, Malander S, Måsbäck A, Hartman L, Nilbert M and Hedenfalk I: Sex steroid hormone receptor expression affects ovarian cancer survival. Transl Oncol 8: 424-433, 2015.

45. Lin SX, Chen J, Mazumdar M, Poirier D, Wang C, Azzi A and Zhou M: Molecular therapy of breast cancer: Progress and future directions. Nat Rev Endocrinol 6: 485-493, 2010.

46. Foster PA, Woo LW, Potter BV, Reed MJ and Purohit A: The use of steroid sulfatase inhibitors as a novel therapeutic strategy against hormone-dependent endometrial cancer. Endocrinology 149: 4035-4042, 2008

47. Chanplakorn N, Chanplakorn P, Suzuki T, Ono K, Chan MS, Miki Y, Saji S, Ueno T, Toi M and Sasano H: Increased estrogen sulfatase (STS) and 17beta-hydroxysteroid dehydrogenase type 1 (17beta-HSD1) following neoadjuvant aromatase inhibitor therapy in breast cancer patients. Breast Cancer Res Treat 120: 639-648, 2010.

48. Day JM, Purohit A, Tutill HJ, Foster PA, Woo LW, Potter BV and Reed MJ: The development of steroid sulfatase inhibitors for hormone-dependent cancer therapy. Ann N Y Acad Sci 1155: 80-87, 2009.

49. Falany JL and Falany CN: Regulation of SULT1E1 expression in Ishikawa adenocarcinoma cells by tibolone. Steroids 71: $880-885,2006$

50. Hormone Health Network: Menopause. http://www.hormone.org/ diseases-and-conditions/womens-health/menopause. Accessed January 17, 2017. 